\title{
Number-phase entanglement and Einstein-Podolsky-Rosen steering
}

\author{
Matteo Fadel $\odot,{ }^{1,2}$ Laura Ares $\odot,{ }^{3}$ Alfredo Luis $\odot,{ }^{3}$ and Qiongyi He $e^{1,4,5, *}$ \\ ${ }^{1}$ State Key Laboratory for Mesoscopic Physics, School of Physics and Collaborative Innovation Center of Quantum Matter, \\ Peking University, Beijing 100871, China \\ ${ }^{2}$ Department of Physics, University of Basel, Klingelbergstrasse 82, 4056 Basel, Switzerland \\ ${ }^{3}$ Departamento de Óptica, Facultad de Ciencias Físicas, Universidad Complutense, 28040 Madrid, Spain \\ ${ }^{4}$ Beijing Academy of Quantum Information Sciences, Beijing 100193, China \\ ${ }^{5}$ Collaborative Innovation Center of Extreme Optics, Shanxi University, Taiyuan, Shanxi 030006, China
}

(Received 22 February 2020; accepted 27 April 2020; published 22 May 2020)

\begin{abstract}
We use the uncertainty relation between the operators associated with the total number of particles and with the relative phase of two bosonic modes to construct entanglement and Einstein-Podolsky-Rosen steering criteria. These can be tested experimentally in a variety of systems, such as optical fields, Bose-Einstein condensates, and mechanical oscillators. While known entanglement criteria involving the phase observable typically require us to perform interference measurements by recombining the two systems, our criteria can be tested through local measurements at two spatially distinct positions to investigate the nonlocal nature of quantum correlations. We present simple examples where our criteria are violated and show their robustness to noise. Apart from being useful for state characterization, they might find application in quantum information protocols, for example, based on number-phase teleportation.
\end{abstract}

DOI: 10.1103/PhysRevA.101.052117

\section{INTRODUCTION}

The Einstein-Podolsky-Rosen (EPR) paradox [1] occurs when measurements on one system allow us to predict measurement results on another system, with an accuracy that beats the limit posed by local uncertainty relations. The observation of such a paradox seems to imply that spatially separated measurements can influence each other irrespective of their separation, a mechanism that Schrödinger called "steering" [2].

From a conceptual point of view, the work by EPR reveals an inconsistency between our idea of local realism and the predictions of quantum mechanics. In the last few decades, EPR steering has motivated numerous fundamental investigations as well as potential applications in quantum technologies $[3,4]$.

Crucially, it has been shown that EPR steering is a distinctive manifestation of quantum correlations that differs from entanglement (state inseparability) [5]. In fact, EPR steering is a form of quantum nonlocality in which the roles of the involved parties are asymmetrical, and it enables the verification of shared entanglement even when one party's measurements are untrusted [6-9]. This has a plethora of applications for one-sided device-independent quantum communication [10-13], as well as for realizing secure quantum teleportation [14-16] and subchannel discrimination [17-19].

A good number of experiments confirming the EPR paradox have been realized for mesoscopic optical fields by $X P$ quadrature measurements $[4,20-25]$. In the case of massive particles, entanglement between two spatially separated mul-

\footnotetext{
*qiongyihe@pku.edu.cn
}

tipartite systems has been demonstrated for atomic ensembles at room temperature [26,27], for Bose-Einstein condensates (BECs) [28-30], and for mechanical oscillators [31,32]. More recently, EPR steering was also observed in BECs [28,29,34].

Criteria to detect entanglement and EPR steering strongly depend on the system (e.g., continuous variable and spin), on the state preparation process, and on the measurement schemes that are available. Here, we will focus on the case of bosonic modes (e.g., optical or atomic), where states can be classified, depending on the preparation processes, as (i) non-number conserving and (ii) number conserving.

Examples of case (i) are the two-mode squeezed states originating from a pair-production process $H / \hbar=\kappa a^{\dagger} b^{\dagger}+$ $\kappa^{*} a b$, where $\langle a b\rangle \neq 0$ but $\left\langle a^{\dagger} b\right\rangle=0$. These are typical states prepared in optics via parametric down-conversion $[3,4]$ or nondegenerate four-wave mixing [33] and in BECs via spinexchanging collisions [34].

On the other hand, examples for case (ii) are states originating from a beam-splitter operation $H / \hbar=\kappa a^{\dagger} b+\kappa^{*} a b^{\dagger}$, so that $\langle a b\rangle=0$ but $\left\langle a^{\dagger} b\right\rangle \neq 0$. These are typical states prepared in optics via linear beam splitters and in double-well BECs through tunneling dynamics [35].

Entanglement and EPR steering can be detected in (i) through criteria based on local measurements of the harmonic oscillator $X P$ quadratures. For mode $a$ these are defined as $X_{A}=\left(a^{\dagger}+a\right) / \sqrt{2}$ and $Y_{A}=\left(a^{\dagger}-a\right) / i \sqrt{2}$, and a similar definition holds for mode $b$. These quadratures are measured experimentally through homodyne detection, where each mode is interfered with a local oscillator that serves as a phase reference. Remarkably, apart from the optical case [20], this has also been demonstrated in atomic [34] systems.

On the other hand, criteria for entanglement and EPR steering based on $X P$ quadrature measurements are not suited 
to states in case (ii) because $\langle a b\rangle=0$. Nevertheless, one can use other criteria, such as the Hillery-Zubairy (HZ) non-Hermitian operator product criterion [36] stating that a violation of $\left|\left\langle a^{\dagger} b\right\rangle\right|^{2} \leqslant\left\langle a^{\dagger} a b^{\dagger} b\right\rangle$ implies that modes $a$ and $b$ are entangled. A generalization of such an inequality can also be used to formulate HZ-type criteria for EPR steering [37,38], confirming that mode $a$ is steered by mode $b$ if $\left|\left\langle a^{\dagger} b\right\rangle\right|^{2}>\left\langle a^{\dagger} a\left(b^{\dagger} b+1 / 2\right)\right\rangle$ or mode $b$ is steered by mode $a$ if $\left.\left|\left\langle a^{\dagger} b\right\rangle\right|^{2}\right\rangle\left\langle\left(a^{\dagger} a+1 / 2\right) b^{\dagger} b\right\rangle$.

However, note that the types of criteria just mentioned require measurements that do not have a clear interpretation in terms of local observables that could be addressed at spatially separated positions $[37,39,40]$. In fact, terms like $\left\langle a^{\dagger} b\right\rangle$ consist of interference measurements that require us to recombine the two modes and are therefore nonlocal measurements. While in many practical situations such measurements can be legitimate for state characterization, in general, they cannot be used to rigorously investigate the nonlocal nature of quantum correlations or for state- or device-independent quantum information tasks.

In this paper we present alternative criteria to detect entanglement and EPR steering between two spatially separated bosonic modes that are based on local measurement of the conjugate number and phase observables. Since the definition of a phase operator in quantum mechanics is notoriously nontrivial [41,42], we pay particular attention to addressing this complication rigorously. In fact, as there is actually no such well-defined operator $[41,43,44]$, we follow the most general quantum description of an observable in terms of a positive operator-valued measure (POVM). Moreover, we quantify the phase uncertainty in terms of the so-called dispersion which, contrary to the variance, is tailored to angular variables. Having these tools defined, we then derive criteria to test entanglement and EPR steering based on the number-phase uncertainty relation $[45,46]$. More specifically, we consider the number sum and phase difference as the two basic compatible observables whose uncertainty is bounded from below for separable or nonsteerable states.

\section{INTUITIVE APPROACH}

Consider two systems, labeled by $j=1,2$, on which measurements $A_{j}$ and $B_{j}$, with $\left[A_{j}, B_{j^{\prime}}\right]=\delta_{j, j} C_{j}$, are performed. For all separable states between the two systems the relation $[47,48]$

$$
\Delta^{2}\left(A_{1}+A_{2}\right)+\Delta^{2}\left(B_{1}-B_{2}\right) \geqslant\left(\left|\left\langle C_{1}\right\rangle\right|+\left|\left\langle C_{2}\right\rangle\right|\right)
$$

is known to hold. Here, $\Delta^{2}(X)=\left\langle X^{2}\right\rangle-\langle X\rangle^{2}$ is the variance of the operator $X$. Similarly, for all nonsteerable states the following relation holds $[3,4]$ :

$$
\Delta^{2}\left(A_{1}+A_{2}\right)+\Delta^{2}\left(B_{1}-B_{2}\right) \geqslant\left|\left\langle C_{2}\right\rangle\right| .
$$

A typical choice of measurements is position and momentum operators, $A_{j}=X_{j}, B_{j}=P_{j}$, for which $C_{j}=i$. This has allowed us to detect entanglement and steering in continuousvariable systems [4,6,20,22-25]. Another possibility is to choose spin observables whose commutator is now also an operator $[49,50]$. This has allowed us to detect entanglement and steering between atomic ensembles [26-30].
When considering bosonic modes, one can also be tempted to choose for $A_{j}$ and $B_{j}$ the particle number operators $N_{j}$ and their conjugate phase operators $\phi_{j}$ (we will discuss the subtleties of the latter in the following paragraph). Naively, these number and phase operators are expected to satisfy the canonical commutation relation $[41,51]$

$$
\left[N_{j}, \phi_{j}\right]=i \quad(\text { in general wrong [41]) }
$$

and therefore to satisfy the uncertainty relation

$$
\Delta^{2} N_{j} \Delta^{2} \phi_{j} \geqslant \frac{1}{4} \quad \text { (in general wrong) }
$$

or, alternatively, as $x^{2}+y^{2} \geqslant 2 \sqrt{x^{2} y^{2}}$,

$$
\Delta^{2} N_{j}+\Delta^{2} \phi_{j} \geqslant 1 \quad \text { (in general wrong). }
$$

From these relations and Eqs. (1) and (2), we are expected to certify entanglement if the inequality

$$
\Delta^{2}\left(N_{1}+N_{2}\right)+\Delta^{2}\left(\phi_{1}-\phi_{2}\right) \geqslant 2
$$

is violated and steering if the inequality

$$
\Delta^{2}\left(N_{1}+N_{2}\right)+\Delta^{2}\left(\phi_{1}-\phi_{2}\right) \geqslant 1
$$

is violated. Note that these criteria involve the total-number operator $N:=N_{1}+N_{2}$ and the phase-difference operator $\phi:=\phi_{1}-\phi_{2}$. These would allow us to detect correlations in observables that are not the usual $X P$ quadratures, therefore characterizing a different class of states.

Unfortunately, as mentioned before, the definition of a phase operator in quantum mechanics is a subtle task, which causes the expressions presented so far to be, in general, wrong $[41,42]$. In fact, while approximate operators satisfying Eqs. (3) and (4) can be found in the limit of small phase fluctuations, this is not true in a more general case. In the following we will treat this problem rigorously to derive entanglement and steering criteria that are valid for arbitrarily large phase fluctuations and that are experimentally practical. As expected, in the limit of small phase fluctuations our criteria allow us to recover Eqs. (6) and (7) from a rigorous framework.

\section{NUMBER-PHASE OBSERVABLES}

In this section we introduce the operators associated with the total number of particles and to the relative phase of two bosonic modes. We discuss their properties, their eigenstates, and how to express their fluctuations. To conclude, we present the uncertainty relation that holds between these observables, which will later be of central importance for deriving entanglement and steering criteria.

\section{A. Number and phase operators}

We are interested in investigating correlations between number and phase observables. In classical physics these two observables arise naturally in the context of, e.g., oscillating fields. In quantum mechanics, however, the definition of a phase operator is less straightforward $[43,44]$.

For a single bosonic mode defined by the operator $a_{j}$, the total number of particles is simply $N_{j}:=a_{j}^{\dagger} a_{j}$. A physically 
meaningful choice is to describe a single-mode phase via the POVM

$$
\Pi_{j}(\phi)=|\phi\rangle_{j}\left\langle\left.\phi\right|_{j},\right.
$$

with the non-normalizable, nonorthogonal phase states

$$
|\phi\rangle_{j}=\frac{1}{\sqrt{2 \pi}} \sum_{n=0}^{\infty} e^{i n \phi}|n\rangle_{j} .
$$

The latter are unit-modulus-eigenvalue eigenstates of the Susskind-Glogower [52] exponential-of-phase operator $E_{j}$, namely, $E_{j}|\phi\rangle_{j}=e^{i \phi}|\phi\rangle_{j}$, such that

$$
E_{j}=\int_{2 \pi} d \phi e^{i \phi} \Pi_{j}(\phi)
$$

For later reference, note that this operator can also be seen as a "normalized" ladder operator, namely, $E_{j}=\sum_{n=0}^{\infty}|n\rangle\langle n+$ $1 \mid=\left(N_{j}+1\right)^{-1 / 2} a_{j}$.

In the following we will be interested in a system constituted by two bosonic modes, defined by the operators $a_{1}$ and $a_{2}$. Inspired by the single-mode case we first define a total-number operator as $N:=N_{1}+N_{2}$. Then, we introduce an operator associated with the relative phase between the two modes, say, $\phi:=\phi_{1}-\phi_{2}$. To this end, let us first construct the joint POVM for the two single-mode phases $\phi_{1}$ and $\phi_{2}$ as

$$
\Pi\left(\phi_{1}, \phi_{2}\right)=\Pi_{1}\left(\phi_{1}\right) \otimes \Pi_{2}\left(\phi_{2}\right) .
$$

Since here we are interested in only the relative phase, we may consider the change in variables

$$
\phi=\phi_{1}-\phi_{2}, \quad \varphi=\phi_{2},
$$

so that

$$
\Pi(\phi, \varphi)=\Pi_{1}(\phi+\varphi) \otimes \Pi_{2}(\varphi) .
$$

From this expression, we finally obtain the POVM associated with the phase difference $\Pi(\phi)$ by integrating out the variable $\varphi$ as

$$
\Pi(\phi)=\int_{2 \pi} d \varphi \Pi(\phi, \varphi)=\int_{2 \pi} d \varphi \Pi_{1}(\phi+\varphi) \otimes \Pi_{2}(\varphi) .
$$

Inserting in this expression Eqs. (8) and (9) for the singlemode phase states and performing the integration over $\varphi$, we arrive at

$$
\Pi(\phi)=\frac{N+1}{2 \pi} \sum_{N=0}^{\infty}|N, \phi\rangle\langle N, \phi|,
$$

where $|N, \phi\rangle$ are now the normalized, nonorthogonal numberphase states

$$
|N, \phi\rangle=\frac{1}{\sqrt{N+1}} \sum_{m=0}^{N} e^{i m \phi}|m\rangle_{1}|N-m\rangle_{2} .
$$

The latter are unit-modulus-eigenvalue eigenstates of the exponential-of-phase-difference operator $E$, namely, $E|N, \phi\rangle=e^{i \phi}|N, \phi\rangle$, such that

$$
E=\int_{2 \pi} d \phi e^{i \phi} \Pi(\phi)=E_{1} E_{2}^{\dagger} .
$$

Moreover, the states $|N, \phi\rangle$ are also eigenstates of the totalnumber operator with eigenvalue $N$. This observation reflects the expected compatibility between total number and phase difference, which means $[N, \Pi(\phi)]=0$. It is interesting to mention that similar to $E_{j}, E$ can be related to the ladder operators $a_{j}$ as $E=\left[\left(N_{1}+1\right) N_{2}\right]^{-1 / 2} a_{1} a_{2}^{\dagger}$ [43]. This relation illustrates how $E$ depends on the coherence between the two bosonic modes.

It is important to emphasize that the relation Eq. (14) expresses the idea that the relative phase $\phi$ between the two modes can be determined via independent local measurements of $\phi_{1}$ and $\phi_{2}$ on the respective mode. This is because Eq. (14) implies that the probability distribution $p(\phi)$ for measuring $\phi$ is obtained from the joint probability distribution $p\left(\phi_{1}, \phi_{2}\right)$ by summing over all configurations for which $\phi_{1}-\phi_{2}=\phi$, namely, we have that

$$
p(\phi)=\int_{2 \pi} d \varphi p\left(\phi_{1}=\phi+\varphi, \phi_{2}=\varphi\right) .
$$

Similarly, Eq. (17) also reflects this fact by showing that the relative phase operator $E$ can be expressed from the single-mode phase operators $E_{1}$ and $E_{2}$. Concretely, the local POVMs associated with the single-mode phases $\Pi_{j}\left(\phi_{j}\right)$ can be implemented experimentally as projective measurements following the prescription given by the Naimark extension [53-55].

In the following, for the sake of readability, we will often call the total-number and phase-difference operators simply number and phase operators.

\section{B. Number variance and phase dispersion}

The formulation of an uncertainty relation between number and phase operators requires a quantification of their fluctuations. For the number observable this is simply achieved by considering the variance

$$
\Delta^{2} N=\left\langle N^{2}\right\rangle-\langle N\rangle^{2} .
$$

However, fluctuations in the phase are not properly characterized by the "standard" definition of variance as in Eq. (19). What is done instead is defining the so-called phase dispersion $D^{2}[45,46,56]$. For a single mode this is computed as

$$
D_{j}^{2}=1-\left|\left\langle E_{j}\right\rangle\right|^{2},
$$

while for two modes the dispersion of the phase difference is (remember $E=E_{1} E_{2}^{\dagger}$ )

$$
D^{2}=1-|\langle E\rangle|^{2} .
$$

While the variance in Eq. (19) is only bounded to be nonnegative, for the dispersion $1 \geqslant D_{j}, D \geqslant 0$ holds, where zero corresponds to no phase fluctuations and unity corresponds to uniform phase distribution. To emphasize the fact that Eq. (20) is associated with the phase of a single mode, while Eq. (21) is associated with the phase difference between two modes, in the following we will call $D_{j}^{2}$ the phase dispersion and $D^{2}$ the relative-phase dispersion.

In the limit of small phase fluctuations, the probability distribution for the phase $P(\phi)$ will peak around some mean value that, without loss of generality, we can consider to be 


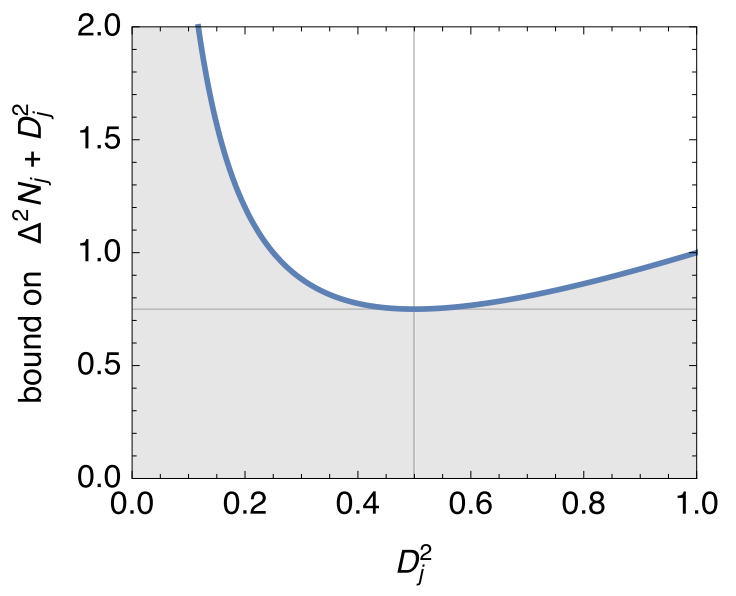

FIG. 1. Evaluation of the bound for $\Delta^{2} N_{j}+D_{j}^{2}$, Eq. (24). The gray region below the blue curve is forbidden by the uncertainty relation. The horizontal gray line at $3 / 4$ indicates the state-independent bound which is tight for $D_{j}^{2}=1 / 2$ (vertical gray line).

zero. Under this limit, a series expansion of the exponentialof-phase operators is valid. Starting from the relation $D^{2}=$ $1-\left|\left\langle e^{i \phi}\right\rangle\right|^{2}$, it is immediately seen that (to second order)

$$
D^{2} \simeq\left\langle\phi^{2}\right\rangle-\langle\phi\rangle^{2} \equiv \Delta^{2} \phi
$$

Here, $\Delta^{2} \phi$ has the usual meaning of variance for the probability distribution for the phase $P(\phi)$.

\section{Number-phase uncertainty relations}

Derivations of entanglement and EPR-steering criteria are often based on the uncertainty relations between the considered observables. For number-phase observables in a single mode we consider the uncertainty relation presented in Ref. [45], which reads

$$
\left(\Delta^{2} N_{j}+\frac{1}{4}\right) D_{j}^{2} \geqslant \frac{1}{4}
$$

This relation can also be written as

$$
\Delta^{2} N_{j}+D_{j}^{2} \geqslant \frac{1}{4 D_{j}^{2}}-\frac{1}{4}+D_{j}^{2} \geqslant \frac{3}{4},
$$

where the constant $3 / 4$ has been found by minimizing the term in the middle (see Fig. 1). Note here that this constant lower bound is, in general, not tight, as it is attained only for a state with $\Delta^{2} N_{j}=1 / 4$ and $D_{j}^{2}=1 / 2$.

In the case of small phase fluctuations we might expect to recover the uncertainty relations (4) and (5). To see that this is the case, we start by rewriting Eq. (23) as

$$
\Delta^{2} N_{j} \frac{D_{j}^{2}}{1-D_{j}^{2}} \geqslant \frac{1}{4} \text {. }
$$

Because for small phase fluctuations $D_{j}^{2} \approx 0$, the series expansion

$$
\frac{D_{j}^{2}}{1-D_{j}^{2}} \simeq D_{j}^{2} \simeq \Delta^{2} \phi_{j}
$$

holds, such that the "naive" uncertainty relation Eq. (4) is recovered. From this and the triangle inequality, we also immediately recover Eq. (5).

To conclude let us remember that, in the case of two modes, total number and phase difference are compatible observables, resulting in a trivial uncertainty relation.

\section{ENTANGLEMENT CRITERION}

Our goal here is to derive an entanglement criterion based on total-number and phase-difference observables. As the associated operators commute, for all quantum states the trivial inequality

$$
\Delta^{2} N+D^{2} \geqslant 0
$$

holds. However, if we restrict ourselves to separable states of the two modes, we are able to provide a nonzero lower bound for $\Delta^{2} N+D^{2}$. The idea behind our proof follows the approach used in Refs. [47,48,57].

In full generality, separable bipartite states can be written as the convex combination

$$
\rho_{\text {sep }}=\sum_{k} p_{k} \rho_{1, k} \otimes \rho_{2, k},
$$

where $p_{k} \geqslant 0, \sum_{k} p_{k}=1$, and $\rho_{j, k}$ is a density matrix for mode $j$. When evaluated on the separable state $\rho_{1, k} \otimes \rho_{2, k}$, the number variance is

$$
\Delta_{k}^{2} N=\Delta_{k}^{2} N_{1}+\Delta_{k}^{2} N_{2} .
$$

For the phase dispersion the decomposition is more subtle. Separability implies $\left|\left\langle E_{1} E_{2}^{\dagger}\right\rangle_{k}\right|^{2}=\left|\left\langle E_{1}\right\rangle_{k}\right|^{2}\left|\left\langle E_{2}^{\dagger}\right\rangle_{k}\right|^{2}=$ $\left|\left\langle E_{1}\right\rangle_{k}\right|^{2}\left|\left\langle E_{2}\right\rangle_{k}\right|^{2}$, and we obtain

$$
D_{k}^{2}=D_{k, 1}^{2}+D_{k, 2}^{2}-D_{k, 1}^{2} D_{k, 2}^{2} .
$$

Using Eqs. (29) and (30), we find that for separable states

$$
\begin{aligned}
\left(\Delta_{k}^{2} N+1\right) D_{k}^{2} & \left(\Delta_{k}^{2} N_{1}+\frac{1}{4}\right) D_{k}^{2}+\left(\Delta_{k}^{2} N_{2}+\frac{1}{4}\right) D_{k}^{2}+\frac{D_{k}^{2}}{2} \\
\geqslant & \frac{1}{2}+\left(\Delta_{k}^{2} N_{1}+\frac{1}{4}\right) D_{k, 2}^{2}\left(1-D_{k, 1}^{2}\right) \\
& +\left(\Delta_{k}^{2} N_{2}+\frac{1}{4}\right) D_{k, 1}^{2}\left(1-D_{k, 2}^{2}\right)+\frac{D_{k}^{2}}{2} \\
\geqslant & \frac{1}{2}+\frac{D_{k, 2}^{2}\left(1-D_{k, 1}^{2}\right)}{4 D_{k, 1}^{2}}+\frac{D_{k, 1}^{2}\left(1-D_{k, 2}^{2}\right)}{4 D_{k, 2}^{2}}+\frac{D_{k}^{2}}{2} \\
= & \frac{1}{2}+\frac{\left(D_{k, 2}^{2}\right)^{2}+\left(D_{k, 1}^{2}\right)^{2}}{4 D_{k, 1}^{2} D_{k, 2}^{2}}+\frac{D_{k, 1}^{2}+D_{k, 2}^{2}-2 D_{k, 1}^{2} D_{k, 2}^{2}}{4} \\
\geqslant & \frac{1}{2}+\frac{2}{4}=1,
\end{aligned}
$$

where, to derive the first two inequalities, we used the uncertainty relation for each system, Eq. (23), and in going to the last line we used the triangle inequality

$$
\left(D_{k, 1}^{2}\right)^{2}+\left(D_{k, 2}^{2}\right)^{2} \geqslant 2 D_{k, 1}^{2} D_{k, 2}^{2}
$$




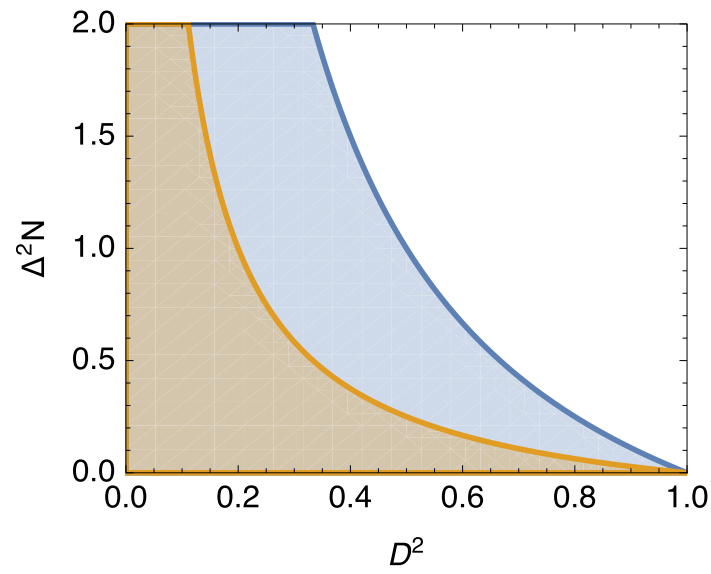

FIG. 2. States in the parameter region below the blue (upper) curve violate inequality Eq. (35), meaning that they are entangled. States in the parameter region below the orange (lower) curve violate (38), meaning that they are two-way steerable.

and

$$
D_{k, 1}^{2}+D_{k, 2}^{2} \geqslant 2 D_{k, 1} D_{k, 2} \geqslant 2 D_{k, 1}^{2} D_{k, 2}^{2} .
$$

Since for all states (28) we have for the variance $\Delta^{2} N \geqslant$ $\sum_{k} p_{k} \Delta_{k}^{2} N$ and for the relative-phase dispersion $D^{2} \geqslant$ $\sum_{k} p_{k} D_{k}^{2}$, we can prove that for all separable states

$$
\begin{aligned}
\left(\Delta^{2} N+1\right) D^{2} & \geqslant\left(\sum_{k} p_{k}\left(\Delta_{k}^{2} N+1\right)\right) \sum_{k} p_{k} D_{k}^{2} \\
& \geqslant\left[\sum_{k} p_{k} \sqrt{\left(\Delta_{k}^{2} N+1\right) D_{k}^{2}}\right]^{2} \\
& \geqslant\left[\sum_{k} p_{k} \sqrt{1}\right]^{2}=1 .
\end{aligned}
$$

Here, in going to the third line we used the Cauchy-Schwarz inequality, and in going to the fourth we used Eq. (31).

To summarize, as we proved that for all separable states

$$
\left(\Delta^{2} N+1\right) D^{2} \geqslant 1
$$

holds, any violation of this inequality certifies entanglement between the two modes. Therefore, Eq. (35) is a bipartite entanglement criterion involving the total number of particles $N$ and the relative-phase dispersion $D^{2}$ associated with the phase difference between the two modes. For illustration purposes, Fig. 2 shows in blue the parameter region for which Eq. (35) is violated. States associated with this region are therefore entangled.

In the limit of small fluctuations in the phase difference, where an expression analogous to Eq. (26) holds for $D^{2}$, we obtain from Eq. (35) the entanglement criterion

$$
\Delta^{2} N \frac{D^{2}}{1-D^{2}} \approx \Delta^{2} N \Delta^{2} \phi \geqslant 1 .
$$

From this, the triangle inequality implies $\Delta^{2} N+\Delta^{2} \phi \geqslant$ $2 \sqrt{\Delta^{2} N \Delta^{2} \phi} \geqslant 2$, which is the entanglement criterion we were expecting for the sum of variances (6).

\section{EINSTEIN-PODOLSKY-ROSEN STEERING CRITERION}

The general idea behind a derivation of an EPR steering criterion follows the same approach as the one for entanglement, but we use the quantum uncertainty relation only for the system that has been assumed to be a "local quantum state" [5]. For the other system (i.e., for the system that steers), we do not assume anything about the variances of the local states except that they are positive.

Inspired by Eq. (31), we start from a similar expression, and using again Eqs. (29) and (30), we find that for all nonsteerable states

$$
\begin{aligned}
\left(\Delta_{k}^{2}\right. & \left.N+\frac{1}{4}\right) D_{k}^{2} \\
& =\left(\Delta_{k}^{2} N_{1}+\Delta_{k}^{2} N_{2}+\frac{1}{4}\right) D_{k}^{2} \\
& =\Delta_{k}^{2} N_{1} D_{k}^{2}+\left(\Delta_{k}^{2} N_{2}+\frac{1}{4}\right)\left(D_{k, 1}^{2}+D_{k, 2}^{2}-D_{k, 1}^{2} D_{k, 2}^{2}\right) \\
& \geqslant \Delta_{k}^{2} N_{1} D_{k}^{2}+\left(\Delta_{k}^{2} N_{2}+\frac{1}{4}\right) D_{k, 1}^{2}\left(1-D_{k, 2}^{2}\right)+\frac{1}{4} \\
& \geqslant \frac{1}{4}
\end{aligned}
$$

where in going to the second to last line we used the uncertainty relation for system 2 (steered party) and in going to the last line we used the fact that for system 1 no uncertainty relation applies, meaning that we can set simultaneously $\Delta_{k}^{2} N_{1}=D_{k, 1}^{2}=0$. Note here that the same result could have been obtained also for the opposite choice in the uncertainty bounds, corresponding to the situation where system 1 is steered.

Following the same steps as in Eq. (34), we find that for all nonsteerable states the following relation holds:

$$
\left(\Delta^{2} N+\frac{1}{4}\right) D^{2} \geqslant \frac{1}{4} .
$$

Therefore, this inequality is a bipartite steering criterion involving the total number of particles $N$ and the relativephase dispersion $D^{2}$, whose violation actually implies twoway steering between the systems.

For illustration purposes, Fig. 2 shows in orange the parameter region for which Eq. (38) is violated. States associated with this region are therefore (two-way) steerable. Moreover, note that Fig. 2 highlights the hierarchy existing between entanglement and steering: steering is a stronger form of correlation, for which entanglement is necessary but not sufficient. As a consequence, every state showing steering is necessarily entangled.

In the limit of small fluctuations in the phase difference, where an expression analogous to Eq. (26) holds for $D^{2}$, we obtain from Eq. (38) the steering criterion

$$
\Delta^{2} N \frac{D^{2}}{1-D^{2}} \approx \Delta^{2} N \Delta^{2} \phi \geqslant \frac{1}{4} .
$$

From this, the triangle inequality implies $\Delta^{2} N+\Delta^{2} \phi \geqslant$ $2 \sqrt{\Delta^{2} N \Delta^{2} \phi} \geqslant 1$, which is the steering criterion we were expecting for the sum of variances (7).

\section{EXAMPLES}

In this section we analyze a number of experimentally relevant examples to illustrate the usefulness of the numberphase entanglement and steering criteria we derived. 


\section{A. Number-phase states}

As a first example we consider the number-phase states described in Eq. (16), for which we have

$$
\Delta^{2} N=0, \quad D^{2}=\frac{2 N+1}{(N+1)^{2}} .
$$

Here, the second equality comes from the fact that $\langle E\rangle=$ $N /(N+1)$. As the total number of particles is constant, both entanglement and EPR criteria, Eqs. (35) and (38), reduce to $D^{2} \geqslant 1$. However, since $0 \leqslant D^{2} \leqslant 1$, the latter coincides with the condition

$$
D^{2}=1
$$

which is violated whenever $N>0$.

From the previous observations, we conclude that when the number of particles is fixed, $\Delta^{2} N=0$, our entanglement and EPR criteria always reduce to $D^{2}=1$ (see Fig. 2). Therefore, every state violating this condition shows directly both entanglement and steering.

\section{B. Split Fock states}

States showing mode entanglement can be prepared using beam splitters. For example, let us consider the input state to be the product state of a Fock state and vacuum, i.e., $|N\rangle|0\rangle$. Then, the output of the beam splitter is $\mathrm{SU}(2)$ coherent states. For a balanced beam splitter where the splitting ratio is 50 : 50 , the output state in the number basis reads

$$
|N, \varphi\rangle=\frac{1}{\sqrt{2^{N}}} \sum_{m=0}^{N} \sqrt{\left(\begin{array}{l}
N \\
m
\end{array}\right)} e^{i m \varphi}|m\rangle|N-m\rangle,
$$

where $\varphi$ is some relative phase introduced by the beam splitter.

These states have $\Delta^{2} N=0$, as the number of particles is set by the input Fock state, while the relative-phase dispersion is

$$
D^{2}=1-\left[\frac{1}{2^{N}} \sum_{m=1}^{N} \sqrt{\left(\begin{array}{l}
N \\
m
\end{array}\right)\left(\begin{array}{c}
N \\
m-1
\end{array}\right)}\right]^{2} .
$$

Like in the previous example, as there are no $N$ fluctuations, entanglement and EPR criteria reduce to $D^{2}=1$, which is violated whenever $N>0$.

For this example it is straightforward to compare our criteria with the HZ-type criteria mentioned in the Introduction [36-38]. A simple calculation yields $\left\langle a^{\dagger} a\right\rangle=\left\langle b^{\dagger} b\right\rangle=N / 2$, $\left|\left\langle a^{\dagger} b\right\rangle\right|^{2}=N^{2} / 4$, and $\left\langle a^{\dagger} a b^{\dagger} b\right\rangle=N(N-1) / 4$. These relations show that the separability criterion $\left|\left\langle a^{\dagger} b\right\rangle\right|^{2} \leqslant\left\langle a^{\dagger} a b^{\dagger} b\right\rangle$ is violated for all $N$, detecting entanglement. On the contrary, the criteria for nonsteerable states, e.g., $\left|\left\langle a^{\dagger} b\right\rangle\right|^{2} \leqslant\left\langle a^{\dagger} a\left(b^{\dagger} b+\right.\right.$ $1 / 2)\rangle$, are never violated. Therefore, this example emphasizes the fact that the number-phase criteria we derived allow us to detect steering in classes of states in which other HZ-type criteria cannot.

\section{Two-mode squeezed states}

Nonclassical states that are known to show entanglement and steering are two-mode squeezed states (TMSSs). These are prepared in optical experiments using parametric downconversion. In the number basis, TMSSs read

$$
|\psi(r)\rangle=\frac{1}{\cosh r} \sum_{m=0}^{\infty}(\tanh r)^{m}|m\rangle|m\rangle,
$$

where $r \geqslant 0$ is a real parameter associated with the squeezing strength. For these states we obtain

$$
\Delta^{2} N=\sinh ^{2}(2 r), \quad D^{2}=1,
$$

implying that both our entanglement and steering criteria, Eqs. (35) and (38), are never violated.

Despite this result, let us remember that TMSSs show a violation of entanglement and steering criteria based on $X P$ quadrature measurements $[3,4]$ which, on the other hand, are useless for number-phase states or split Fock states. Therefore, this example emphasizes the fact that the number-phase criteria we derived allow us to detect entanglement and steering in classes of states in which standard $X P$ criteria cannot.

\section{States with noise in the total number}

So far we have discussed pure states, which represent idealized situations. In fact, experiments always deal with different types of noise. We analyze here the effect of an imperfect state preparation resulting in shot-to-shot fluctuations in the total number of particles.

The expectation value of an operator $A$ is

$$
\langle A\rangle=\sum_{N=0}^{\infty} \operatorname{tr}\left(\rho_{N} A\right),
$$

where $\rho_{N}$ is the restriction of $\rho$ to the subspace with total number $N$.

To give a concrete example, consider a statistical mixture of number-phase states (16). [The same results have been obtained numerically for the split Fock states (42).] We have $\rho_{N}=p(N)|N, \phi\rangle\langle N, \phi|$, where $p(N)=\operatorname{tr}\left(\rho_{N}\right)$ is the probability of the total number to be $N$. The variance of $N$ is directly obtained from $p(N)$, while the phase dispersion is obtained from

$$
\langle E\rangle=\sum_{N=0}^{\infty} p(N) \frac{N}{N+1} .
$$

Let us consider three possibilities for the probability distribution $p(N)$ : Poissonian, Gaussian, and thermal.

Poissonian statistics. This is the case of typical number fluctuations of coherent sources where the number variable is distributed as

$$
p(N)=\frac{\bar{N}^{N}}{N !} e^{-\bar{N}},
$$

where $\bar{N}$ is the mean number of particles. For this statistics we obtain

$$
\Delta^{2} N=\bar{N}, \quad D^{2}=1-\left(\frac{\bar{N}-1+e^{-\bar{N}}}{\bar{N}}\right)^{2},
$$

resulting in no violation of the criteria (35) and (38) for all $\bar{N}$.

Gaussian statistics. This is the case of large enough number of photons that $N$ can be treated as a continuous variable 
obeying Gaussian statistics

$$
p(N)=\frac{1}{\sqrt{2 \pi} \Delta N} \exp \left[-\frac{(N-\bar{N})^{2}}{2 \Delta^{2} N}\right],
$$

where $\bar{N}$ is the mean number of particles and we assume $\Delta N \ll \bar{N}$. In this limit, to obtain simple expressions, let us consider a series expansion of $N /(N+1)$ in Eq. (47) around $\bar{N}$ to get

$$
\frac{N}{N+1} \simeq \frac{\bar{N}}{\bar{N}+1}+\frac{N-\bar{N}}{(\bar{N}+1)^{2}}-\frac{(N-\bar{N})^{2}}{(\bar{N}+1)^{3}},
$$

so that after replacing Eq. (47) by an integral we get

$$
\langle E\rangle \simeq \int_{-\infty}^{\infty} d N p(N) \frac{N}{N+1} \simeq \frac{\bar{N}}{\bar{N}+1}-\frac{\Delta^{2} N}{(\bar{N}+1)^{3}} .
$$

Furthermore, since this approximation is valid provided $\bar{N} \gg$ 1 , we may also consider a series expansion in powers of $1 / \bar{N}$ to get to first order

$$
D^{2} \simeq \frac{2}{\bar{N}}
$$

In this case the violation of the criteria (35) and (38) depends on the specific values of $\bar{N}$ and $\Delta N$. For

$$
\bar{N} \gg 1
$$

we observe that entanglement is revealed by Eq. (35) when $\Delta^{2} N<\bar{N} / 2$, while steering is revealed by Eq. (38) when $\Delta^{2} N<\bar{N} / 8$. Note here that having $\Delta^{2} N<\bar{N}$ corresponds to sub-Poissonian statistics.

Thermal statistics. This is the typical case of number fluctuations resulting from thermal light sources, for which

$$
p(N)=\frac{1}{\bar{N}+1}\left(\frac{\bar{N}}{\bar{N}+1}\right)^{N},
$$

where $\bar{N}$ is again the mean number while the variance in this case is

$$
\Delta^{2} N=\bar{N}(\bar{N}+1) .
$$

The relative-phase dispersion is directly evaluated from

$$
\langle E\rangle=\sum_{N=0}^{\infty} p(N) \frac{N}{N+1}=1-\frac{\ln (\bar{N}+1)}{\bar{N}} .
$$

Also in this case we have that there is no violation of the criteria (35) and (38) for any $\bar{N}$.

\section{CONCLUSIONS}

In this work we presented criteria to detect entanglement and EPR steering between two bosonic modes that are based on number and phase measurements. To achieve this, we first presented the operators associated with the latter measurements, together with their uncertainty relation. In particular, to describe fluctuations in the phase we used the concept of dispersion, which is an analog of the variance for cyclic variables. Then, inspired by the typical EPR sum and difference quadratures, we derived entanglement and steering criteria in terms of the total number of particles and relative phase measurements. These are inequivalent to the typical criteria based on $X P$ quadrature measurements, and therefore, they allow us to detect quantum correlations in classes of states that are, in general, different. Moreover, in contrast to criteria requiring us to access interference terms such as $\left\langle a^{\dagger} b\right\rangle$, the criteria we presented can be tested by performing local measurements, allowing us to explore the nonlocal character of quantum correlations. We gave a few examples where our criteria are violated, showing that they are useful in concrete experimental scenarios, for example involving optical modes, double-well BECs or mechanical oscillators. Apart from being of fundamental interest, our results could also find application in quantum information protocols, such as number-phase teleportation [58-62].

\section{ACKNOWLEDGMENTS}

M.F. acknowledges support from the National Natural Science Foundation of China (Grants No. 11622428 and No. 61675007) and from the Swiss National Science Foundation. Q.H. is thankful for the support from the National Natural Science Foundation of China (Grants No. 61675007 and No. 11975026), Beijing Natural Science Foundation (Grant No. Z190005), and the Key R\&D Program of Guangdong Province (Grant No. 2018B030329001). L.A. and A.L. acknowledge financial support from Spanish Ministerio de Economía y Competitividad Project No. FIS2016-75199-P. L.A. acknowledges financial support from the European Social Fund and the Spanish Ministerio de Ciencia Innovación y Universidades, Contract Grant No. BES-2017-081942.
[1] A. Einstein, B. Podolsky, and N. Rosen, Can quantummechanical description of physical reality be considered complete? Phys. Rev. 47, 777 (1935).

[2] E. Schrödinger, Discussion of probability relations between separated systems, Math. Proc. Cambridge Philos. Soc. 31, 555 (1935); Probability relations between separated systems, 32, 446 (1936).

[3] M. D. Reid, Demonstration of the Einstein-Podolsky-Rosen paradox using nondegenerate parametric amplification, Phys. Rev. A 40, 913 (1989).

[4] M. D. Reid, P. D. Drummond, W. P. Bowen, E. G. Cavalcanti, P. K. Lam, H. A. Bachor, U. L. Andersen, and G. Leuchs, Colloquium: The Einstein-Podolsky-Rosen paradox: From concepts to applications, Rev. Mod. Phys. 81, 1727 (2009), and experiments referenced therein.

[5] H. M. Wiseman, S. J. Jones, and A. C. Doherty, Steering, Entanglement, Nonlocality, and the Einstein-Podolsky-Rosen Paradox, Phys. Rev. Lett. 98, 140402 (2007); S. J. Jones, H. M. Wiseman, and A. C. Doherty, Entanglement, EinsteinPodolsky-Rosen correlations, Bell nonlocality, and steering, Phys. Rev. A 76, 052116 (2007).

[6] V. Händchen, T. Eberle, S. Steinlechner, A. Samblowski, T. Franz, R. F. Werner, and R. Schnabel, Observation of 
one-way Einstein-Podolsky-Rosen steering, Nat. Photonics 6, 596 (2012).

[7] E. G. Cavalcanti, M. J. W. Hall, and H. M. Wiseman, Entanglement verification and steering when Alice and Bob cannot be trusted, Phys. Rev. A 87, 032306 (2013).

[8] D. Cavalcanti and P. Skrzypczyk, Quantum steering: A review with focus on semidefinite programming, Rep. Prog. Phys. 80, 024001 (2017).

[9] R. Uola, A. C. S. Costa, H. C. Nguyen, and O. Gühne, Quantum steering, Rev. Mod. Phys. 92, 015001 (2020).

[10] M. Tomamichel and R. Renner, Uncertainty Relation for Smooth Entropies, Phys. Rev. Lett. 106, 110506 (2011).

[11] C. Branciard, E. G. Cavalcanti, S. P. Walborn, V. Scarani, and H. M. Wiseman, One-sided device-independent quantum key distribution: Security, feasibility, and the connection with steering, Phys. Rev. A 85, 010301 (2012).

[12] T. Gehring, V. Händchen, J. Duhme, F. Furrer, T. Franz, C. Pacher, R. F. Werner, and R. Schnabel, Implementation of continuous-variable quantum key distribution with composable and one-sided-device-independent security against coherent attacks, Nat. Commun. 6, 8795 (2015).

[13] N. Walk, S. Hosseini, J. Geng, O. Thearle, J. Y. Haw, S. Armstrong, S. M. Assad, J. Janoušek, T. C. Ralph, T. Symul, H. M. Wiseman, and P. K. Lam, Experimental demonstration of Gaussian protocols for one-sided device-independent quantum key distribution, Optica 3, 634 (2016).

[14] M. D. Reid, Signifying quantum benchmarks for qubit teleportation and secure quantum communication using EinsteinPodolsky-Rosen steering inequalities, Phys. Rev. A 88, 062338 (2013).

[15] Q. He, L. Rosales-Zárate, G. Adesso, and M. D. Reid, Secure Continuous Variable Teleportation and Einstein-PodolskyRosen Steering, Phys. Rev. Lett. 115, 180502 (2015).

[16] C.-Y. Chiu, N. Lambert, T.-L. Liao, F. Nori, and C.-M. Li, Nocloning of quantum steering, npj Quantum Inf. 2, 16020 (2016).

[17] M. Piani and J. Watrous, Necessary and Sufficient Quantum Information Characterization of Einstein-Podolsky-Rosen Steering, Phys. Rev. Lett. 114, 060404 (2015).

[18] S.-L. Chen, C. Budroni, Y.-C. Liang, and Y.-N. Chen, Natural Framework for Device-Independent Quantification of Quantum Steerability, Measurement Incompatibility, and Self-Testing, Phys. Rev. Lett. 116, 240401 (2016).

[19] K. Sun, X.-J. Ye, Y. Xiao, X.-Y. Xu, Y.-C. Wu, J.-S. Xu, J.-L. Chen, C.-F. Li, and G.-C. Guo, Demonstration of EinsteinPodolsky-Rosen steering with enhanced subchannel discrimination, npj Quantum Inf. 4, 12 (2018).

[20] Z. Y. Ou, S. F. Pereira, H. J. Kimble, and K. C. Peng, Realization of the Einstein-Podolsky-Rosen paradox for continuous variables, Phys. Rev. Lett. 68, 3663 (1992).

[21] J.-C. Lee, K.-K. Park, T.-M. Zhao, and Y.-H. Kim, EinsteinPodolsky-Rosen Entanglement of Narrow-Band Photons from Cold Atoms, Phys. Rev. Lett. 117, 250501 (2016).

[22] Q. Y. He and M. D. Reid, Quantifying the Mesoscopic Nature of Einstein-Podolsky-Rosen Nonlocality, Phys. Rev. Lett. 123, 120402 (2019), and experiments referenced therein.

[23] S. Armstrong, M. Wang, R. Y. Teh, Q. H. Gong, Q. Y. He, J. Janousek, H. A. Bachor, M. D. Reid, and P. K. Lam, Multipartite Einstein-Podolsky-Rosen steering and genuine tripartite entanglement with optical networks, Nat. Phys. 11, 167 (2015).
[24] X. W. Deng, Y. Xiang, C. Tian, G. Adesso, Q. Y. He, Q. H. Gong, X. L. Su, C. D. Xie, and K. C. Peng, Demonstration of Monogamy Relations for Einstein-Podolsky-Rosen Steering in Gaussian Cluster States, Phys. Rev. Lett. 118, 230501 (2017).

[25] Y. Cai, Y. Xiang, Y. Liu, Q. He, and N. Treps, Versatile multipartite Einstein-Podolsky-Rosen steering via a quantum frequency comb, arXiv:1910.13698.

[26] H. Krauter, C. A. Muschik, K. Jensen, W. Wasilewski, J. M. Petersen, J. I. Cirac, and E. S. Polzik, Entanglement Generated by Dissipation and Steady State Entanglement of Two Macroscopic Objects, Phys. Rev. Lett. 107, 080503 (2011).

[27] B. Julsgaard, A. Kozhekin, and E. S. Polzik, Experimental long-lived entanglement of two macroscopic objects, Nature (London) 413, 400 (2011).

[28] M. Fadel, T. Zibold, B. Decamps, and P. Treutlein, Spatial entanglement patterns and Einstein-Podolsky-Rosen steering in Bose-Einstein condensates, Science 360, 409 (2018).

[29] P. Kunkel, M. Prüfer, H. Strobel, D. Linnemann, A. Frölian, T. Gasenzer, M. Gärttner, and M. K. Oberthaler, Spatially distributed multipartite entanglement enables EPR steering of atomic clouds, Science 360, 413 (2018).

[30] K. Lange, J. Peise, B. Lücke, I. Kruse, G. Vitagliano, I. Apellaniz, M. Kleinmann, G. Toth, and C. Klempt, Entanglement between two spatially separated atomic modes, Science 360, 416 (2018).

[31] R. Riedinger, A. Wallucks, I. Marinković, C. Löschnauer, M. Aspelmeyer, S. Hong, and S. Gröblacher, Remote quantum entanglement between two micromechanical oscillators, Nature (London) 556, 473 (2018).

[32] C. F. Ockeloen-Korppi, E. Damskägg, J.-M. Pirkkalainen, M. Asjad, A. A. Clerk, F. Massel, M. J. Woolley, and M. A. Sillanpää, Stabilized entanglement of massive mechanical oscillators, Nature (London) 556, 478 (2018).

[33] R. E. Slusher, L. W. Hollberg, B. Yurke, J. C. Mertz, and J. F. Valley, Observation of Squeezed States Generated by FourWave Mixing in an Optical Cavity, Phys. Rev. Lett. 55, 2409 (1985); M. D. Reid and D. F. Walls, Squeezing in nondegenerate four-wave mixing, Phys. Rev. A 33, 4465 (1986).

[34] J. Peise, I. Kruse, K. Lange, B. Lücke, L. Pezzé, J. Arlt, W. Ertmer, K. Hammerer, L. Santos, A. Smerzi, and C. Klempt, Satisfying the Einstein-Podolsky-Rosen criterion with massive particles, Nat. Commun. 6, 8984 (2015).

[35] J. Estève, C. Gross, A. Weller, S. Giovanazzi, and M. K. Oberthaler, Squeezing and entanglement in a Bose-Einstein condensate, Nature (London) 455, 1216 (2008).

[36] M. Hillery and M. S. Zubairy, Entanglement Conditions for Two-Mode States, Phys. Rev. Lett. 96, 050503 (2006).

[37] Q. Y. He, P. D. Drummond, M. K. Olsen, and M. D. Reid, Einstein-Podolsky-Rosen entanglement and steering in twowell Bose-Einstein-condensate ground states, Phys. Rev. A 86, 023626 (2012).

[38] E. G. Cavalcanti, Q. Y. He, M. D. Reid, and H. M. Wiseman, Unified criteria for multipartite quantum nonlocality, Phys. Rev. A 84, 032115 (2011).

[39] G. Toth, C. Simon, and J. I. Cirac, Entanglement detection based on interference and particle counting, Phys. Rev. A 68, 062310 (2003).

[40] I. Urizar-Lanz and G. Toth, Number-operator-annihilationoperator uncertainty as an alternative for the number- 
phase uncertainty relation, Phys. Rev. A 81, 052108 (2010).

[41] P. Carruthers and M. M. Nieto, Phase and angle variables in quantum mechanics, Rev. Mod. Phys. 40, 411 (1968); M. M. Nieto, Quantum phase and quantum phase operators: Some physics and some history, Phys. Scr. T48, 5 (1993), and references therein.

[42] R. Lynch, The quantum phase problem: A critical review, Phys. Rep. 256, 367 (1995).

[43] A. Luis and L. L. Sánchez-Soto, Phase-difference operator, Phys. Rev. A 48, 4702 (1993).

[44] A. Luis and L. L. Sánchez-Soto, Quantum phase difference, phase measurements and Stokes operators, Prog. Opt. 41, 421 (2000).

[45] T. Opatrný, Number-phase uncertainty relations, J. Phys. A 28, 6961 (1995).

[46] T. Opatrný, Mean value and uncertainty in optical phase, a simple mechanical analogy, J. Phys. A 27, 7201 (1994).

[47] L.-M. Duan, G. Giedke, J. I. Cirac, and P. Zoller, Inseparability Criterion for Continuous Variable Systems, Phys. Rev. Lett. 84, 2722 (2000).

[48] R. Simon, Peres-Horodecki Separability Criterion for Continuous Variable Systems, Phys. Rev. Lett. 84, 2726 (2000).

[49] W. P. Bowen, N. Treps, R. Schnabel, and P. K. Lam, Experimental Demonstration of Continuous Variable Polarization Entanglement, Phys. Rev. Lett. 89, 253601 (2002).

[50] V. Giovannetti, S. Mancini, D. Vitali, and P. Tombesi, Characterizing the entanglement of bipartite quantum systems, Phys. Rev. A 67, 022320 (2003).
[51] P. A. Dirac, The fundamental equations of quantum mechanics, Proc. R. Soc. London 109, 642 (1925).

[52] L. Susskind and L. Glogower, Quantum mechanical phase and time operator, Phys. Phys. Fiz. 1, 49 (1964).

[53] M. A. Naimark, On spectral functions of a symmetric operator, Izv. Akad. Nauk SSSR, Ser. Mat. 7, 285 (1943).

[54] K. L. Pregnell and D. T. Pegg, Single-Shot Measurement of Quantum Optical Phase, Phys. Rev. Lett. 89, 173601 (2002).

[55] N. Dalla Pozza and M. G. A. Paris, Naimark extension for the single-photon canonical phase measurement, Phys. Rev. A 100, 032126 (2019).

[56] E. Breitenberger, Uncertainty measures and uncertainty relations for angle observables, Found. Phys. 15, 353 (1985).

[57] H. F. Hofmann and S. Takeuchi, Violation of local uncertainty relations as a signature of entanglement, Phys. Rev. A 68, 032103 (2003).

[58] G. J. Milburn and S. L. Braunstein, Quantum teleportation with squeezed vacuum states, Phys. Rev. A 60, 937 (1999).

[59] S. Yu and C.-P. Sun, Canonical quantum teleportation, Phys. Rev. A 61, 022310 (2000).

[60] P. T. Cochrane, G. J. Milburn, and W. J. Munro, Teleportation using coupled oscillator states, Phys. Rev. A 62, 062307 (2000)

[61] P. T. Cochrane and G. J. Milburn, Teleportation with the entangled states of a beam splitter, Phys. Rev. A 64, 062312 (2001).

[62] N.-K. Tran and O. Pfister, Quantum teleportation with close-tomaximal entanglement from a beam splitter, Phys. Rev. A 65, 052313 (2002). 